\title{
Neuro-Oncology
}

\author{
LETTER TO THE EDITOR
}

\section{The Autopsy: Obtaining Quality Assurance of Treatment}

\author{
M. Shahriar Salamat
}

\section{Dear Editor:}

I read with great interest the thoughtful letter by Dr. Kendra Peterson that appeared in the January 2001 issue of Neuro-Oncology. While we hope that one day all of our patients with CNS tumors will be cured, we must not lose sight that still much is to be learned about these tumors and their therapies. In this respect, the ultimate method of quality assurance, the autopsy, should not be forgotten.

Regrettably, in recent years, there seems to have been an inverse relation between multiple methods of imaging, biopsying and resecting, classifying and grading, and treating CNS tumors and the percentage of tumor patients who end up having an autopsy. For most of us, experience in postmortem evaluation of treated CNS tumors can only be summarized in anecdotal recounts, rather than through experience with this additional scientific approach that can determine the effects of therapies that we provide. A real understanding of tumor response to therapy is achieved only if we all make a concerted effort to obtain autopsy permits for our terminal tumor patients. The success rate of the attainment of autopsies for patients with neurodegenerative diseases and the development of multiple neurodegenerative brain tissue banks in this country and around the world are testaments to the effectiveness of protocols and educational materials for caregivers who come in contact with these patients. We need to develop guidelines, some to be built into therapeutic protocols, that allow for a more systematic and successful approach to requesting an autopsy when the end nears for our CNS tumor patients.

M. Shahriar Salamat, M.D., Ph.D.

Neuropathology

University of Wisconsin Hospital

Madison, WI 53792

Neuro-Oncology 3, 215, 2001 (Posted to Neuro-Oncology [serial online], Doc. 01-020, May 14, 2001. URL $<$ neuro-oncology.mc.duke.edu>) 